\title{
Diaphragmatic hernia as an early ultrasound manifestation of Apert syndrome
}

\author{
Przemysław Kosiński, Katarzyna Luterek, Mirosław Wielgoś \\ $1^{\text {st }}$ Department of Obstetrics and Gynecology, Medical University of Warsaw, Poland
}

Apert syndrome, or acrocephalosyndactyly (type 1), is a congenital anomaly of a genetic origin, with an estimated birth prevalence of 1 in 65000 live births. It occurs as a result of heterozygous mutations in the fibroblast growth factor receptor 2 (FGFR2) gene located at 10q26. The diagnosis is usually based on the physical features, but molecular analysis of FGFR2 may be very helpful in uncertain cases. The most common clinical presentation consists in coronal craniosynostosis, skin syndactyly of the hands ("mitten glove") and feet, and craniofacial dysmorphic features. However, Apert syndrome may also be associated with some other rare anomalies, e.g. mid-facial hypoplasia, strabismus, pulmonary atresia, ventriculomegaly, corpus callosum defects, spinal fusion, tracheoesophageal fistula, pyloric stenosis, polycystic kidneys, bicornuate uterus, and cryptorchidism.

We present a case of Apert syndrome associated with congenital diaphragmatic hernia. To the best of our knowledge, only four such cases have been reported so far in the literature. The patient was diagnosed with isolated congenital diaphragmatic hernia at the routine anomaly scan at 22 weeks. Amniocentesis confirmed normal karyotype without any structural chromosomal abnormalities. The patient was referred to the $1^{\text {st }}$ Department of Obstetrics and Gynecology. Detailed ultrasound scan revealed mild ventriculomegaly and an unusual shape of the cavum septum pellucidum. Fetal MRI scan was performed at 23 weeks and no abnormalities were detected. The next ultrasound scan again confirmed an abnormal shape of the posterior ventricles coexisting with unusual shape of the cavum septum pellucidum. The patient was recommended to have another MRI scan of the fetal brain after 26 weeks of gestation. The MRI scan yet again confirmed normal structures of the fetal brain and no other abnormalities of fetal anatomy were detected in either scan. Due to severe congenital diaphragmatic hernia, the patient had FETO (Fetoscopic Endotracheal Occlusion) at 27 weeks. The procedure itself and the postoperative time were both uneventful. She had follow-up scans every two weeks to evaluate fetal growth, amniotic fluid volume and response to the tracheal occlusion. At 30-31 weeks, the abnormal shape of the fetal head and hands became apparent. The fetal scull had a rectangular-like shape compared to the ultrasound performed at 22 weeks (Fig. 1 and 2). At 32-33 weeks, just before fetoscopy for balloon retrieval, an ultrasound scan of the fetal profile (broad forehead, mid-facial hypoplasia, hypertelorism, exophthalmos) and hands ("mitten-like" hands) was highly suggestive for Apert syndrome (Fig. 3 and 4). The patient was informed about high risk of genetic syndrome in her baby. The second fetoscopy and the balloon retrieval was successful. The patient had premature rapture of membranes and the child was born via the vaginal route at 36 weeks of gestation. Apert syndrome was confirmed due to baby's phenotype. The baby died one hour after the delivery due to cardiovascular and respiratory distress.

Invasive treatment in prenatal medicine is offered to fetuses without genetic syndromes. However, some rare genetic disorders cannot be detected in routine karyotyping or ultrasound. Congenital diaphragmatic hernia is a severe and complex condition, which has already been described in connection with Apert syndrome. This coexistence is extremely rare and this case describes diaphragmatic hernia as the early ultrasound manifestation of Apert syndrome.

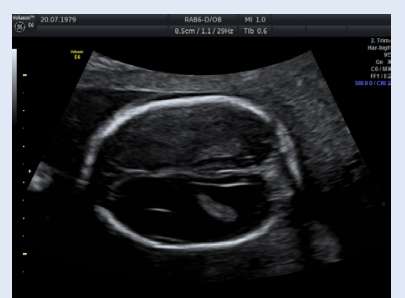

Figure 1. Cross section of fetal head and brain at 20 weeks of gestation

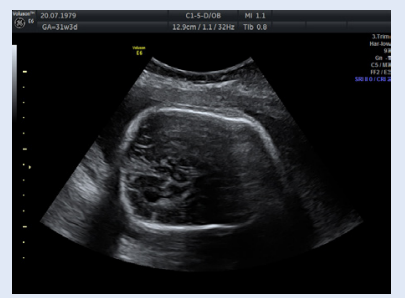

Figure 2. Abnormal shape of fetal head at 31 weeks of gestation

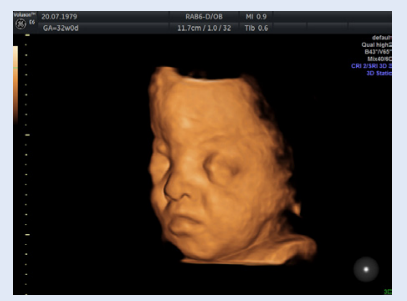

Figure 3. Facial features of Apert syndrome (mid-facial hypoplasia, hypertelorism, exophthalmos)

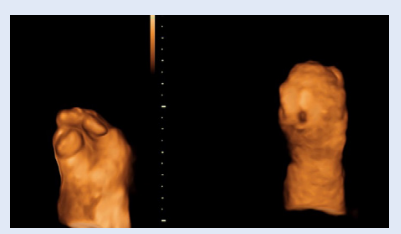

Figure 4. Abnormal hands ("mitten-like" hands) in a fetus with Apert syndrome 